\title{
Correction to: Near Real Time Satellite Event Detection, Characterization, and Operational Assessment Via the Exploitation of Remote Photoacoustic Signatures
}

\author{
Justin Spurbeck ${ }^{1}$ - Moriba K. Jah ${ }^{1}$ - Daniel Kucharski ${ }^{2}$ - James C. S. Bennett ${ }^{3}$. \\ James G. Webb ${ }^{4}$
}

Published online: 7 June 2021

(C) American Astronautical Society 2021

Correction to: The Journal of the Astronautical Sciences (2021) 68:197-224 https://doi.org/10.1007/s40295-021-00252-5

This article was updated to replace an incorrect version that was published due to an error in the production process.

The online version of the original article can be found at https://doi.org/10.1007/s40295-021-00252-5

Justin Spurbeck

jspurbeck@utexas.edu

Moriba K. Jah

moriba@utexas.edu

1 Aerospace Engineering and Engineering Mechanics Department, Cockrell School of Engineering, The University of Texas at Austin, Austin, TX 78712, USA

2 Research Fellow, Space Environment Research Centre, SERC, Canberra, Australia. Visiting Researcher, Institute for Computational Engineering and Sciences, The University of Texas at Austin, Austin, TX 78712, USA

3 Astrodynamics Group Leader, EOS Space Systems Pty Ltd, Queanbeyan, Australia. Research Program Leader, Space Environment Research Center, Canberra, Australia

4 Instrument Scientist, EOS Space Systems Pty Ltd, Queanbeyan, Australia 